\title{
Field dependence of the adiabatic temperature change in second order phase transition materials: Application to Gd
}

\author{
V. Franco, ${ }^{1, a)}$ A. Conde, ${ }^{1}$ J. M. Romero-Enrique, ${ }^{2}$ Y. I. Spichkin, ${ }^{3}$ V. I. Zverev, ${ }^{4}$ and \\ A. M. Tishin ${ }^{4}$ \\ ${ }^{1}$ Dpto. Física de la Materia Condensada, ICMSE-CSIC, Universidad de Sevilla, P.O. Box 1065, 41080 \\ Sevilla, Spain \\ ${ }^{2}$ Dpto. Física Atómica, Molecular y Nuclear, Área de Física Teórica, Universidad de Sevilla, P.O. Box \\ 1065, 41080 Sevilla, Spain \\ ${ }^{3}$ Advanced Magnetic Technologies and Consulting, Ltd., Promishlennaya st. 4, Troitsk, Moscow region \\ 142190, Russia \\ ${ }^{4}$ Moscow State University, Physics Faculty, Leninskie Gory, Moscow 119991, Russia
}

(Received 13 August 2009; accepted 10 October 2009; published online 19 November 2009)

\begin{abstract}
The field dependence of the adiabatic temperature change $\Delta T_{\mathrm{ad}}$ of second order phase transition materials is studied, both theoretically and experimentally. Using scaling laws, it is demonstrated that, at the Curie temperature, the field dependence of $\Delta T_{\mathrm{ad}}$ is characterized by $H^{1 / \Delta}$. Therefore, as the magnetic entropy change $\Delta S_{M}$ follows a $H^{(1-\alpha) / \Delta}$ power law, these two dependencies coincide only in the case of a mean field model. A phenomenological construction of a universal curve for $\Delta T_{\mathrm{ad}}$ is presented, and its theoretical justification is also given. This universal curve can be used to predict the response of materials in different conditions not available in the laboratory (extrapolations in field or temperature), for enhancing the resolution of the data and as a simple screening procedure for the characterization of materials. (c) 2009 American Institute of Physics. [doi:10.1063/1.3261843]
\end{abstract}

\section{INTRODUCTION}

The optimization of magnetic materials to be applied in magnetic refrigerator prototypes goes through the understanding of the magnetic field dependence of their magnetocaloric effect, in order to fine tune the response of the material under the applied field available in the actual device. Recently, there has been an increasing interest in describing the field dependence of the magnetic entropy change, $\Delta S_{M}$, but much less efforts have been made to analyze the evolution of the adiabatic temperature change, $\Delta T_{\text {ad }}$, as the field is increased. This paper studies in detail the similarities and differences between the field dependencies of these magnitudes, in order to be able to use this knowledge for practical applications.

It has been recently shown that there exists a universal curve for the magnetic entropy change, $\Delta S_{M}$, of second order phase transition materials. It can be constructed using a phenomenological procedure ${ }^{1}$ which does not require the knowledge of either the equation of state or the critical exponents of the material. This construction facilitates the application of the universal curve for practical purposes for the characterization of materials, like predicting the response of the material under experimental conditions not available in the laboratory (extrapolations in temperature or field), enhancing the resolution of the experimental data, etc. It has been demonstrated that it can be successfully applied to different families of soft magnetic amorphous alloys ${ }^{1,2}$ (for which it was initially developed) and subsequently extended to rare earth based crystalline materials. ${ }^{3-5}$ The theoretical background for this universal curve construction lies on scaling laws. ${ }^{6}$ More

${ }^{a)}$ Electronic mail: vfranco@us.es. recently, it has been shown ${ }^{7,8}$ that the same phenomenological procedure is suitable for scaling the adiabatic temperature change, $\Delta T_{\mathrm{ad}}$, of second order phase transition materials. However, the justification for that finding relied only on a strong simplification: either a weak dependence of the specific heat with magnetic field, which allows to reduce the description of the field dependence of $\Delta T_{\text {ad }}$ to that of $\Delta S_{M}$, or the assumption that as the specific heat scales with field close to the Curie transition, the adiabatic temperature change should also scale.

The aim of this work is to show that it is possible to use scaling laws to predict the existence of the universal curve for $\Delta T_{\mathrm{ad}}$ and that, in contrast with the previous literature which restrict the approach to a mean field model, the field dependence of $\Delta T_{\mathrm{ad}}$ is not necessarily the same as that of $\Delta S_{M}$ (which is the prediction of the mean field model). Polycrystalline Gd has been used to evidence the accuracy of these claims.

\section{EXPERIMENTAL}

Polycrystalline Gd (99.5 at. \% pure) was obtained from a commercial source. The method of the direct $\Delta T_{\text {ad }}(H)$ measurements was described in detail by Tishin et al. ${ }^{9}$ and Spichkin et al. ${ }^{10}$ The measurements were made on the Magnetocaloric Measuring Setup "MagEq MMS 801" manufactured by Advanced Magnetic Technologies and Consulting, Ltd., Moscow, Russia. The magnetic field was created by permanent magnet Halbach magnetic field source with changeable magnetic field in its working bore. $\Delta T$ values were measured by a differential thermocouple with the measuring junction clamped between two pieces of the material under investigation and a reference junction placed on the nonmagnetic me- 




FIG. 1. (Color online) Temperature dependence of the adiabatic temperature change measured in polycrystalline $\mathrm{Gd}$ for maximum applied fields ranging from $0.38 \times 10^{6}$ up to $1.50 \times 10^{6} \mathrm{~A} / \mathrm{m}$. Lines are spline fits to the data.

tallic sample holder near the sample. $\Delta T$ and $H$ values were recorded simultaneously and continuously over the whole cycle of the magnetic field change, which makes it possible to construct the $\Delta T_{\text {ad }}(H)$ curves and on the basis of this data get $\Delta T_{\mathrm{ad}}(T)$ at necessary magnetic field change $(\Delta H)$ value. The measurements were made near the corresponding Curie temperatures $T_{C}$ at the magnetic field change rate of $1 \mathrm{~T} / \mathrm{s}$. The maximum magnetic field $(H)$ was $1.87 \mathrm{~T}$.

\section{RESULTS AND DISCUSSION}

Figure 1 shows the different $\Delta T_{\mathrm{ad}}(T)$ curves for different values of the maximum applied field. In order to find the position and magnitude of the peak, a spline interpolation has been performed and is indicated as continuous lines in the figure. At the peak, the field dependence of $\Delta T_{\mathrm{ad}}$ can be assumed to be a power law, with an exponent $p$

$$
\Delta T_{\mathrm{ad}}^{\mathrm{pk}} \propto H^{p}
$$

in an analogous way to that of the power law for $\Delta S_{M}$. From the interpolated curves of Fig. 1, the exponent $p$ can be extracted by performing a nonlinear fit to the data, as shown in Fig. 2. The obtained value is $p=0.70 \pm 0.01$. This result is in good agreement with previous literature results. ${ }^{11}$ Two conclusions can be extracted from this value: the exponent is different from that of $\Delta S_{M}$ for $\mathrm{Gd}^{4}{ }^{4}$ and it is different from the mean field prediction of $2 / 3$ of the mean field model. ${ }^{12}$

The adiabatic temperature change can be expressed as

$$
\Delta T_{\mathrm{ad}}=-\mu_{0} \int_{0}^{H} \frac{T}{c_{p}}\left(\frac{\partial M}{\partial T}\right)_{H} d H .
$$

According to Krasnow and Stanley, ${ }^{13}$ the specific heat scales with field as

$$
\frac{c_{p}(t, H)}{H^{-\alpha / \Delta}}=c\left(\frac{H}{|t|^{\Delta}}\right)
$$

where $c$ is a scaling function. The scaling form of the magnetic equation of state can be expressed as

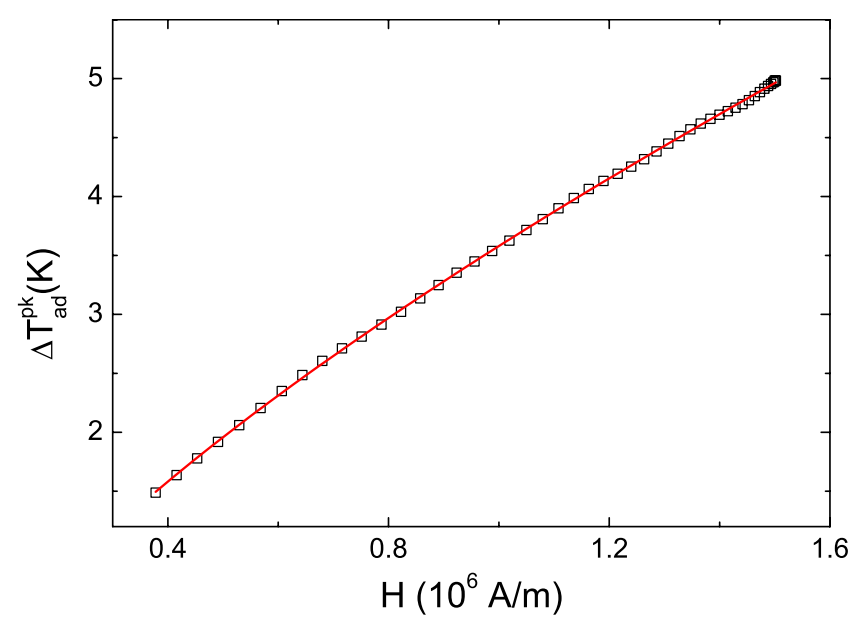

FIG. 2. (Color online) Field dependence of the maximum adiabatic temperature change determined from the spline fits of Fig. 1. Line is the fit to a power law.

$$
\frac{M}{|t|^{\beta}}=m_{ \pm}\left(\frac{H}{|t|^{\Delta}}\right)
$$

where $t=\left(T-T_{C}\right) / T_{C}$ is the reduced temperature, $T_{C}$ is the Curie temperature, $\Delta=\beta \delta$ is the gap exponent and the plus (minus) sign corresponds to $t>0(t<0)$, respectively. Therefore, Eq. (2) transforms to

$$
\begin{aligned}
\Delta T_{\text {ad }} & =-\mu_{0} \int_{0}^{H /|t|^{\Delta}} d x \frac{T}{H^{-\alpha / \Delta} c(x)}|t|^{1-\alpha}\left[\beta m_{ \pm}(x)-\Delta x m_{ \pm}^{\prime}(x)\right] \\
& =-\mu_{0}|t| \int_{0}^{H /|t|^{\Delta}} d x \frac{T}{c(x)} x^{\alpha / \Delta}\left[\beta m_{ \pm}(x)-\Delta x m_{ \pm}^{\prime}(x)\right] \\
& =|t| \widetilde{f}\left(t / H^{1 / \Delta}\right) \\
& =H^{1 / \Delta} f\left(t / H^{1 / \Delta}\right) .
\end{aligned}
$$

This equation shows that if the reduced temperature $t$ is rescaled by a factor proportional to $H^{1 / \Delta}$, and the adiabatic temperature change also by $H^{1 / \Delta}$, the experimental data should collapse onto the same curve. As $\Delta T_{\text {ad }}$ is a temperature magnitude, it is reasonable that it scales with field in the same way as the temperature axis. It is worth noting the difference with the scaling for the magnetic entropy change, which is $H^{(1-\alpha) / \Delta}$ (where $1-\alpha=\beta+\Delta-1$ ). ${ }^{6}$ In the case of the mean field model, $\alpha=0$ and the field dependences for both magnitudes coincide, as predicted in Ref. 12. However, for any other value set of the critical exponents, the field dependences of $\Delta T_{\text {ad }}$ and $\Delta S_{M}$ are different, in agreement with our experimental results.

Taking into account the small values of the critical exponent $\alpha$, a fact which introduces an additional complication for its experimental determination, the comparison between the two different field dependencies of $\Delta T_{\text {ad }}$ and $\Delta S_{M}$ could be a method for determining the value of exponent $\alpha$. However, as the experimental setups for measuring the magnetization curves, from which $\Delta S_{M}$ is calculated, and the setup for measuring $\Delta T_{\text {ad }}$ are intrinsically different, the finding of minor differences in both field dependencies relies extensively on the calibration in field and temperature of both 




FIG. 3. (Color online) Universal curve for the adiabatic temperature change curves of Fig. 1. Line is the average of the different curves corresponding to the different fields.

setups. Moreover, magnetometers usually do not place the temperature sensor in contact with the sample or sample holder, but measure the temperature of the sample chamber. Therefore, depending on measuring conditions, small oscillations of sample temperature could be expected, imposing a limitation on the accurate determination of $\alpha$ by using this comparison. More detailed studies on these experimental constraints are being undertaken.

In order to construct the phenomenological universal curve for $\Delta T_{\mathrm{ad}}$, an analogous procedure to that described in Refs. 1 and 6 for $\Delta S_{M}$ can be used. It consists in normalizing the $\Delta T_{\text {ad }}$ curves with respect to their maximum and rescaling the temperature axis as

$$
\theta=\left(T-T_{C}\right) /\left(T_{r}-T_{C}\right),
$$

where $T_{r}$ is the temperature of the point of each curve which fulfils $\Delta T_{\mathrm{ad}} / \Delta T_{\mathrm{ad}}^{\mathrm{pk}}=K$ for $T>T_{C}$, and $K$ is a constant smaller than one which can be arbitrarily chosen for each study (for the present work $K=0.5$ ) and whose purpose is to set the equivalent points of the different curves at the same reduced temperature $\theta=1$. Figure 3 shows the universal curve constructed for the experimental $\Delta T_{\mathrm{ad}}(H, T)$ curves of Fig. 1. The agreement between this phenomenological construction and the prediction of Eq. (5) can be checked by testing the field dependence of the reference temperature $T_{r}$. According to the previously presented theoretical predictions, $T_{r}$ should scale with field in the same way as $\Delta T_{\text {ad }}$ does. Therefore, once the field dependence of $\Delta T_{\mathrm{ad}}$ has been extracted from the nonlinear fit of Fig. 2, it has been used to construct the abscissa axis of Fig. 4, where the straight line is a linear fit to the data, showing a good agreement between theory and phenomenological procedure. A further test would be to check that the temperature at which the peak of $\Delta T_{\mathrm{ad}}$ takes place, $T_{\mathrm{pk}}$, also scales with field in the same way. In this case, the procedure is trickier, as for determining $T_{\mathrm{pk}}$ with enough resolution the previously mentioned spline interpolation of the curves has to be performed. By imposing a resolution of 0.1 $\mathrm{K}$ in the interpolate temperature axis, Fig. 5 shows the evolution of the peak position with field, together with a linear fit to the data. Regardless of the discretization of the data

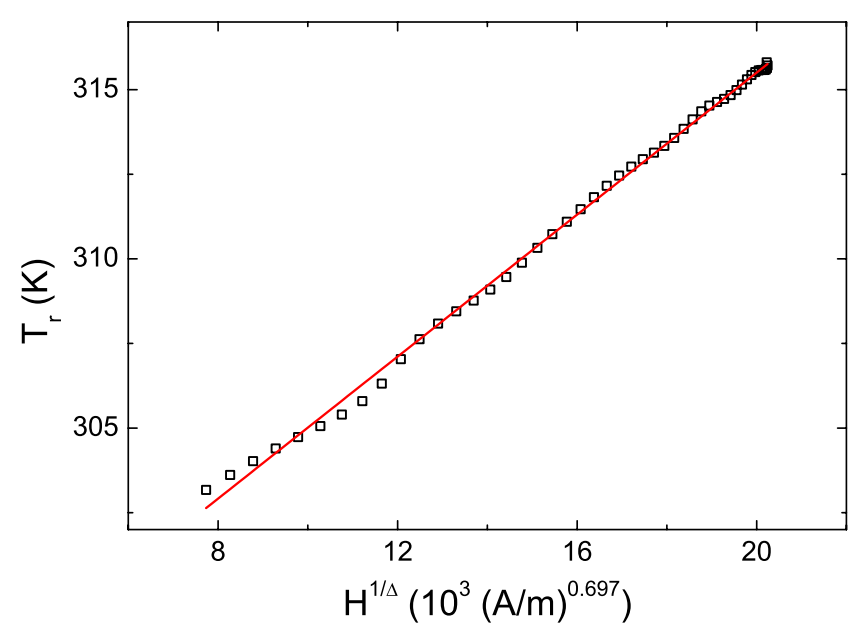

FIG. 4. (Color online) Field dependence of the reference temperature used for constructing the universal curve. The exponent $\Delta$ was obtained from the nonlinear fit of Fig. 2. Line corresponds to a linear fit to the data.

points imposed by the resolution, the predicted field dependence is fulfilled. For the maximum applied field of $1.87 \mathrm{~T}$, the increase of the peak temperature with applied magnetic field remains around $1 \mathrm{~K}$. The intercept of the linear fits of Figs. 4 and 5 should give the Curie temperature of the material and should be coincident in both cases. The values of the intercepts are $294.5 \pm 0.1$ and $294.3 \pm 0.1$, respectively, confirming the good agreement between the experimental results and the theoretical predictions. The accurate determination of the Curie temperature of the sample could be done using standard methods as that of Belov-Goryaga or the one proposed by Kouvel-Fisher. Less standard techniques should be tested on raw samples.

There would be another possible test of the accuracy of the claims, alternative to the self consistent test presented above. It would consist in using the literature values of the critical exponents of Gd for checking the field dependencies of the peak adiabatic temperature change and reference temperatures. However, there is a large dispersion in the literature data for $\mathrm{Gd}$, ascribed to different sample preparations,

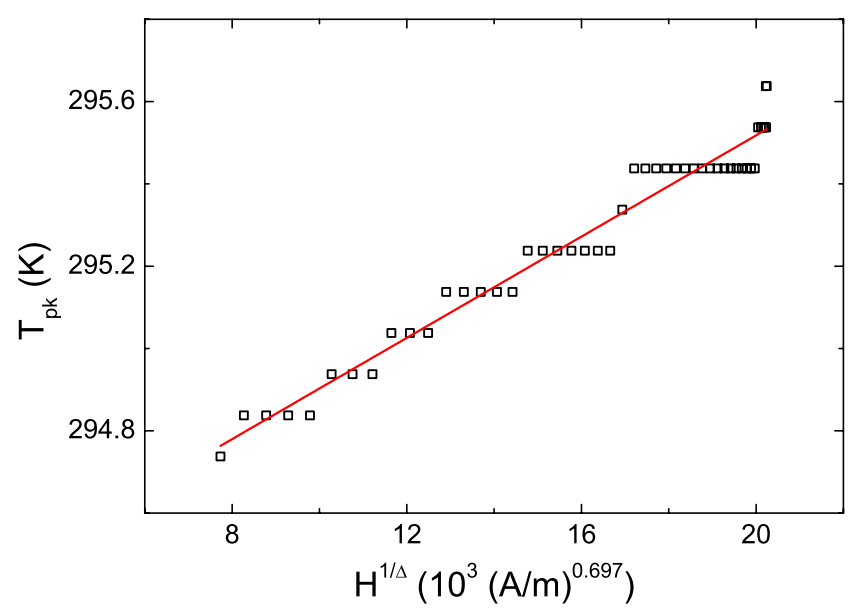

FIG. 5. (Color online) Temperature of the maximum adiabatic temperature change, determined from the spline fits of Fig. 1, as a function of field. The exponent $\Delta$ was obtained from the nonlinear fit of Fig. 2. Line corresponds to a linear fit to the data. 
different purity, etc. and results would not be conclusive. Therefore, we consider that checking that all the magnitudes scale with field in the proper way, for the same sample and the same set of measurements, is a stronger test of the consistency of the results.

\section{CONCLUSIONS}

A physical basis for the phenomenological construction of the universal curve for $\Delta T_{\text {ad }}$ has been given by using scaling laws. It is shown that the field dependence of $\Delta T_{\mathrm{ad}}$ and $\Delta S_{M}$ at the temperature of the peak are not the same, except in the case of a mean field model. This difference in the scaling exponent for both magnitudes can be used to determine the critical exponent $\alpha$. The phenomenologically constructed universal curve for $\Delta T_{\text {ad }}$ and the presented field dependencies can be used for predicting the response of the material in different conditions not available in the laboratory (extrapolations in field or temperature), for enhancing the resolution of the data and as a simple screening procedure for the characterization of materials. Theoretical predictions have been checked using experimental result for pure $\mathrm{Gd}$.

\section{ACKNOWLEDGMENTS}

This work was supported by the Spanish Ministry of Science and Innovation and EU FEDER (Grant No. MAT 2007-65227), and the PAI of the Regional Government of Andalucía (Grant No. P06-FQM-01823). Y. I. Spichkin and
V. I. Zverev thank Advanced Magnetic Technologies and Consulting Ltd. for financial support. Authors are thankful to I. Zubkov for the assistance with experimental measurements.

${ }^{1}$ V. Franco, J. S. Blázquez, and A. Conde, Appl. Phys. Lett. 89, 222512 (2006)

${ }^{2}$ V. Franco, J. S. Blázquez, M. Millán, J. M. Borrego, C. F. Conde, and A. Conde, J. Appl. Phys. 101, 09C503 (2007).

${ }^{3}$ V. Franco, A. Conde, V. K. Pecharsky, and K. A. Gschneidner, Jr., Europhys. Lett. 79, 47009 (2007).

${ }^{4}$ Q. Y. Dong, H. W. Zhang, J. R. Sun, B. G. Shen, and V. Franco, J. Appl. Phys. 103, 116101 (2008).

${ }^{5}$ V. Franco, R. Caballero-Flores, A. Conde, Q. Y. Dong, and H. W. Zhang, J. Magn. Magn. Mater. 321, 1115 (2009).

${ }^{6}$ V. Franco, A. Conde, J. M. Romero-Enrique, and J. S. Blázquez, J. Phys.: Condens. Matter 20, 285207 (2008).

${ }^{7}$ Y. I. Spichkin, I. Zubkov, V. Franco, and A. M. Tishin, 3rd International Conference of IIR on Magnetic Refrigeration at Room Temperature, Des Moines, IA, 11-15 May 2009, pp. 217-221.

${ }^{8}$ Tishin A.M., Franco V., Conde A., Zubkov I., Spichkin Yu., The method of determination of the temperature and field dependences of the adiabatic temperature change with the help of the universal curve. Application for the patent of Russian Federation 2009117924/28(024684), date of application 14.05.2009.

${ }^{9}$ A. M. Tishin, K. A. Gschneidner, Jr., V. K. Pecharsky, and Y. I. Spichkin, Second IIF-IIR International Conference on Magnetic Refrigeration at Room Temperature, Portoroz, Slovenia, 11-13 April 2007, pp. 35-45.

${ }^{10}$ Y. I. Spichkin, A. M. Tishin, D. B. Kopeliovich, and A. Y. Malyshev, 3rd International Conference of IIR on Magnetic Refrigeration at Room Temperature, Des Moines, IA, 11-15 May 2009, pp. 173-180.

${ }^{11}$ V. K. Pecharsky and K. A. Gschneidner, Jr., Int. J. Refrig. 29, 1239 (2006).

${ }^{12}$ H. Oesterreicher and F. T. Parker, J. Appl. Phys. 55, 4334 (1984).

${ }^{13}$ R. Krasnow and H. E. Stanley, Phys. Rev. B 8, 332 (1973). 